\title{
Targeted Agents and Resistance Mechanism in Renal Cell Cancer
}

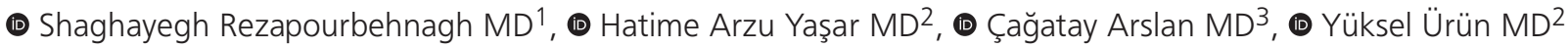 \\ ${ }^{1}$ Ankara University Institute of Biotechnology, Department of Biotechnology, Ankara, Turkey \\ 2Ankara University Faculty of Medicine, Department of Medical Oncology, Ankara, Turkey \\ 3 Bahçeşehir University Faculty of Medicine, Department of Internal Medicine and Medical Oncology, Istanbul, Turkey
}

\begin{abstract}
Renal cell carcinoma (RCC) exhibits multidrug resistance protein P-glycoprotein expression due to the proximal tubular origin and in this regard, it is resistant to a large number of cytotoxic chemotherapy. The identification of the molecular pathogenesis, genetics, and epigenetics of RCC has led to new target points such as vascular endothelial growth factor. Tyrosine kinase inhibitors have been used mainly for treatment, but recently, immune checkpoint inhibitors have also been used in the treatment of RCC. Despite these treatments, response rates are not sufficient in the majority of patients. Primary resistance or acquired resistance to the treatment might be seen. Defining these resistance mechanisms will contribute to the management of the treatment and will help in to identify new treatment targets. In this review, we focus on the molecular mechanisms and resistance mechanisms of targeted-therapy.
\end{abstract}

Keywords: Renal cell carcinoma, resistance mechanisms, tyrosine kinase inhibitors,

\section{Introduction}

Renal cell carcinoma (RCC) emerges from renal tubular epithelial cells. Among newly diagnosed cancers, it is the $6^{\text {th }}$ most common cancer in men (5\%) and $10^{\text {th }}$ in women (3\%) (1). The most common subtypes are clear cell RCC (ccRCC) (75\%), papillary RCC (10\%) and chromophobe RCC (5\%) (2). cCRCCs are the most common cause of RCC-related mortality (3). In the treatment of metastatic RCC ( $\mathrm{mRCC})$, targeted therapies have significantly improved the management of the disease in the last in last ten years. Agents that target vascular endothelial growth factor (VEGF) and mammalian target of Rapamycin (mTOR) pathways have proven to be effective in the treatment of $\mathrm{mRCC}$, mainly in cCRCC. Recent immunotherapy studies with planned death-1 (PD-1) receptors and ligands (PD-L1) and "immune control point" targeted inhibitors such as cytotoxic T-lymphocyte antigen-4 (CTLA-4) have changed the current practice.

In order to increase the efficacy of targeted therapies and to obtain more pronounced survival results, the molecular pathogenesis of RCC cases should be known in more detail and the prognosis of patients should be determined according to molecular characteristics. Through molecular biology research, RCCs have been identified at genetic and epigenetic levels, from single nucleotide polymorphisms to large chromosome defects (4).

\section{Discussion}

\section{Molecular Pathogenesis of Renal Cell Carcinoma}

In ccRCCs, von Hippel-Lindau (VHL) tumor suppressor gene loss is observed and changes in the genes involved in the chromatin remodeling complex are detected. Several studies have shown the association of ccRCC to lysine (K)-specific demethylase (KDM6A, KDM5C), histone methyltransferase SETD2 (suppressor of variegation, Enhancer of zeste, Trithorax-domain containing 2) and polybromo1 (PBRM1) genes in the chromatin remodeling complex (5).

The VHL tumor suppressor gene (TSG) is one of the earliest identified genes in the $3 p 25$ locus related to $C C R C C$, and is present in $64-100 \%$ of cCRCC tumors as a driver mutation. The leading cause of death in $75 \%$ of patients with $V H L$ syndrome is ccRCC. $V H L$ protein (pVHL) binds hypoxia-induced factor $\alpha(H I F \alpha)$ and induces ubiquitin-mediated proteolysis (6). Inactivation of the $\mathrm{VHL}$ gene formed by mutation, deletion or methylation leads to the accumulation of HIF $\alpha$ under normal oxygen conditions without hypoxia, and promotes tumor growth. Genetic changes in the VHL gene are believed to be 
related only to $\operatorname{ccRCC}$, however, van Houwelingen et al. (7) identified VHL gene mutations in $15 \%$ of non-ccRCCs in their study on a group of sporadic RCC patients in the Netherlands. However, in other studies, no mutation in the VHL gene was observed in other RCC subtypes $(8,9)$.

The discovery of the VHL gene in familial and sporadic cCRCC has revolutionized the treatment of advanced RCC. Treatments aimed at suppressing angiogenesis by VEGF or platelet-derived growth factor (PDGF) mediated pathways have replaced immunotherapies such as interferon alpha (IFN $\alpha$ ) and interleukin-2 (IL-2), which are used in the treatment of mRCC. Tyrosine kinase inhibitors (TKIs) (axitinib, cabozantinib, lenvatinib, pazopanib, sorafenib and sunitinib), VEGF monoclonal antibody (bevacizumab) and mTOR inhibitors (everolimus and temsirolimus) have received FDA approval as targeted treatment drugs for RCC (10).

In cCRCC, the VHL gene is not the only genetic deviation in the chromosome $3 p$ region. After the VHL gene, the mutation in the gene of Fragile histidine triad protein (FHIT) found in the region $3 p 14.2$ was observed in about $40 \%$ of the cases. The chromosomal translocation $t(3 ; 8)$ (p14.2; q24) was first identified in hereditary RCC and was then found in sporadic cCRCC as a widespread loss site in chromosome 3 (11). The specific function of the FHIT protein is still unclear. Another TSC associated with $c \mathrm{RCC}$ in the $3 p$ chromosomal region is the Ras association domain family 1 isoform A (RASSF1A) gene found in 3p21.3. RASSF1A protein regulates microtubule formation, cell cycle control and apoptosis (12). The RASSF1A promoter region becomes inactive by hypermethylation and is frequently seen in ovarian, breast and lung cancers besides CCRCC (13). RASSF1A inactivation is present in approximately $44 \%$ of papillary RCCs (14). Recently, next-generation sequencing or exon sequencing studies have shown several new genes related to chromatin modification in cCRCCs (15). Newly defined genes are PBRM1, AT-rich interaction domain 1A (ARID1A), BRCA1 associated protein-1 (BAP1), SETD2, and lysine-specific demethylase 5C (KDM5C) (16). PBRM1 mutations were detected in $41 \%$ of cCRCCs. All PBRM1, BAP1 and SETD2 genes are described to be inactivated in a similar manner to VHL near-3p21 region by Knudson's two-hit hypothesis (17).

DNA sequence analysis, transcriptome and integrated data analysis in recent studies have revealed frequently mutated signaling pathways such as phosphatidylinositol 3-kinase (PI3K)AKT-mTOR and p53 in ccRCC $(18,19)$. PI3K-AKT-mTOR pathway regulating angiogenesis, cell cycle progression and proliferation is the target of mTOR inhibitors (temsirolimus and everolimus). mTOR, phosphatase and tensin analog (PTEN), PIK3CA, AKT2 and other genes in this pathway have been shown to be mutated in $26-28 \%$ of ccRCC tumors.

\section{Molecular Pathways in Targeted Treatment of Renal Cell Carcinoma}

After understanding the molecular pathogenesis of RCCs, targeted therapies have been developed. In the treatment of RCC, inhibition of signaling pathways that play an important role in carcinogenesis is targeted. Research has shown that angiogenesis is excessive in RCC. Due to these results, the inhibition of the angiogenesis pathway is prominent in targeted therapies. The most important gene associated with angiogenesis in RCC is the VHL gene. This has led to the use of agents that primarily target the VHL/HIF pathway in the treatment of RCC.

Anti-angiogenic agents targeting VEGF and mTOR pathways have proven their efficacy in the treatment of mRCC. Recently, immunotherapy with CTLA-4 and PD-1 receptors and "checkpoint" targeted inhibitors such as PD-L1 has been included in the treatment of RCC. Numerous clinical studies, particularly combination therapies, continue.

\section{VEGF Inhibitors}

VEGF-tyrosine kinase receptor inhibitors (axitinib, pazopanib, sorafenib, sunitinib) block the signaling pathway by binding to the intracytoplasmic region of the receptor. Pazopanib is an angiogenesis inhibitor that acts by VEGF receptor (VEGFR) $-1,-2$ and -3 , and PDGF receptor (PDGFR) $\alpha, \beta$ and c-kit. In phase III study, pazopanib was compared with placebo, and the progression-free survival (PFS) was longer (9.2 months vs. 4.2 months) and the objective response rate (ORR) was higher $(30 \%$ vs. $3 \%)$ in the pazopanib group (20). In the updated results of survival and safety analysis, the reason why pazopanib treatment did not show a survival advantage was attributed to the permission to pass from placebo group to pazopanib group after disease progression (21).

Sunitinib is a multi-target tyrosine kinase inhibitor acting via VEGFR-1, -2, and -3, PDGF-R $\alpha$ and $\beta$, c-kit, FLT-3, colony stimulating factor receptor (CSF-1R), and neurotrophic factor receptor (RET). In phase III study, sunitinib and IFN $\alpha$ were compared in the first-line treatment. The PFS was longer (11 months vs. 6 months, respectively) and ORR was higher (34\% vs. $6 \%$, respectively) with sunitinib treatment. Sunitinib was approved by the FDA for the first-line treatment of mRCC (22).

Both agents showed similar efficacy in phase III COMPARZ study, a non-inferiority study comparing sunitinib with pazopanib $(23,24)$.

Bevacizumab is a monoclonal antibody that binds to circulating VEGF $A$ with high affinity and inhibits signal transduction. In the phase III AVOREN study, the combination of IFN $\alpha$ and bevacizumab was compared with the combination of IFN $\alpha$ and placebo, and PFS in the bevacizumab group was approximately five months longer (10.2 months vs. 5.4 months) (25).

Sorafenib, similar to sunitinib, have inhibitory effects on VEGF, PDGF-R, c-kit and c-MET. In the comparative study, the median PFS was 5.5 months with sorafenib and 2.8 months in the placebo group. Overall survival (OS) was 17.8 months in the sorafenib group and 14.3 months in the placebo group $(\mathrm{p}=0.0287)(26)$.

Different current treatment options and their rankings in $\mathrm{mRCC}$ treatment are summarized in Table 1 and Table 2.

In the AXIS study comparing axitinib with sorafenib in the second line treatment of $\mathrm{MRCC}$, higher ORR and longer PFS were shown in the axitinib group (27). Afterwards, the efficacy of axitinib in the first line treatment was investigated. In a study comparing axitinib treatment with sorafenib in the first line treatment, PFS was 10.1 months in the axitinib group and 6.5 months in the sorafenib group (28). 
Cabozantinib is a newly developed, powerful VEGF-2 and c-MET dual inhibitor. Recent studies have shown that MET signals are important in sustaining VEGF signals, tumor angiogenesis, proliferation and patient survival (29). These results also indicate that MET signaling may play a role in VEGF inhibitor resistance. In the METEOR study comparing cabozantinib with everolimus in previously treated patients, cabozantinib has been shown to contribute to PFS for approximately four months (7.4 months vs. 3.8 months) (30) and to OS for approximately five months (21.4 months vs. 16.5 months) (31). Dovitinib is a new TKI that targets both VEGF and fibroblast growth factor receptor (FGFR) pathways. Preclinical studies have also shown that this dual-acting TKI has activity against topoisomerase (32). In the phase III study, median PFS with dovitinib and sorafenib treatments were found to be 3.7 and 3.6 months, respectively, and dovitinib did not show an additional contribution to clinical benefit compared to sorafenib treatment. In a phase II study comparing lenvatinib, the combination of lenvatinib and everolimus and everolimus alone, the combination therapy showed PFS and OS advantage against everolimus alone (PFS: 14.6 months vs 5.5 months; OS: 25.5 months vs 15.4 months) $(33,34)$ and was included in the post-first line treatment sequence.

Table 1. mRCC treatment options (clear cell carcinoma dominant)

\begin{tabular}{|l|l|l|}
\hline & First-line & Second-line \\
\hline $\begin{array}{l}\text { Tyrosine kinase } \\
\text { inhibitor }\end{array}$ & $\begin{array}{l}\text { Axitinib } \\
\text { Cabozantinib } \\
\text { Pazopanib } \\
\text { Sunitinib }\end{array}$ & $\begin{array}{l}\text { Axitinib } \\
\text { Cabozantinib } \\
\text { Lenvatinib + everolimus } \\
\text { Pazopanib } \\
\text { Sorafenib } \\
\text { Sunitinib }\end{array}$ \\
\hline $\begin{array}{l}\text { VEGF receptor } \\
\text { antibody }\end{array}$ & Bevacizumab + IFNa2b & Bevacizumab \\
\hline mTOR inhibitor & Temsirolimus & Everolimus Temsirolimus \\
\hline Immunotherapy & $\begin{array}{l}\text { HD-IL2 } \\
\text { Nivolumab }\end{array}$ & $\begin{array}{l}\text { Nivolumab } \\
\text { HD-IL2 }\end{array}$ \\
\hline
\end{tabular}

VEGF: Vascular endothelial growth factor, mTOR: Mammalian target of rapamycin, HD-IL2: High-dose interleukin-2, IFNa2b: Interferon alpha and interleukin-2, MRCC: Metostatic renal cell carcinoma

Table 2. mRCC treatment options (sub-types except for clear cell carcinoma)

\begin{tabular}{|l|l|}
\hline Tyrosine Kinase Inhibitor & $\begin{array}{l}\text { Axitinib } \\
\text { Cabozantinib } \\
\text { Lenvatinib + everolimus } \\
\text { Pazopanib } \\
\text { Sorafenib } \\
\text { Sunitinib }\end{array}$ \\
\hline VEGF receptor antibody & $\begin{array}{l}\text { Bevacizumab } \\
\text { Bevacizumab + erlotinib } \\
\text { Bevacizumab + everolimus }\end{array}$ \\
\hline mTOR inhibitor & $\begin{array}{l}\text { Everolimus } \\
\text { Temsirolimus }\end{array}$ \\
\hline Immunotherapy & Nivolumab \\
\hline $\begin{array}{l}\text { mRCC: Metastatic renal cell carcinoma, VEGF: Vascular endothelial growth } \\
\text { factor, mTOR: Mammalian target of rapamycin }\end{array}$ \\
\hline
\end{tabular}

\section{mTOR Inhibitors}

The second important treatment target is the mTOR pathway. The PI3K/AKT/mTOR pathway plays an important role in many cancers (35). The binding of VEGF to the VEGF receptor causes activation of the mTOR pathway. Activation of this pathway provides cell growth, proliferation, angiogenesis, mobility and survival function, as well as activating protein synthesis and transcription in the cell. Active mTOR pathway accelerates the translation of both ribosomal protein and tumor progression factors, as well as activation of HIF and cell cycle regulators. In addition, the PI3K/AKT/mTOR pathway was modified in $28 \%$ of tumors (36). Blocking the mTOR signal leads to a reduction of protein translation, angiogenesis and inhibition of tumor cell proliferation. The mTOR protein consists of mTOR complex 1 (mTORC1) and mTOR complex 2 (mTORC2) found in the cytoplasm. This complex acts as a regulator in processes such as cell metabolism, growth and proliferation. In targeted therapies, mTOR inhibitors inhibit mTORC1 only. Drug resistance resulting from treatment with temsirolimus or everolimus develops as a result of mTORC2 activation in the background (37).

Temsirolimus blocks mTORC1 by forming a complex with FK-506 binding protein (FKBP) in mRCC (37).

In phase III ARCC study comparing temsirolimus with IFN therapy in first-line treatment in patients with poor prognosis in mRCC, temsirolimus treatment has been shown to statistically significantly contribute to survival (10.9 months in temsirolimus and 7.3 months in IFN groups) (38).

Comparing sorafenib and temsirolimus as second-line treatment after sunitinib, the benefit of temsirolimus treatment in improving PFS or OS has not been shown (39).

Everolimus is a mTOR inhibitor used in second- or third-line therapy in $\mathrm{mRCC}$ that progresses after VEGF-TKI treatment (40).

\section{Immunotherapy}

Prior to 2005, cytokines (INF $\alpha$ and IL2) were the most effective treatment agents used alone or in combination for the treatment of mRCC. High-dose interleukin-2 (HD-IL2) was the only treatment option for long-term response to mRCC treatment. Long-term responses can be obtained with HD-IL2 in $5-7 \%$ of patients. These therapies have given way to less toxic and more effective TKI treatments. Combination of IFN $\alpha$ with bevacizumab is among the recommended treatment options for $\mathrm{mRCC}$.

CTLA-4 inhibitors (Ipilimumab) and PD-1/PD-L1 inhibitors (Nivolumab) have been used in clinical practice in the treatment of $\mathrm{mRCC}$ (41).

In phase III CheckMate 025 study comparing nivolumab with everolimus in second-line treatment of $\mathrm{mRCC}$, nivolumab was shown to contribute approximately 5.4 months to survival (25 months vs. 19.6 months) (42) and it has taken its place in the second line treatment. In the CheckMate 214 study, the combination of nivolumab and ipilimumab was compared with sunitinib in the first-line treatment. The median overall survival could not be achieved with nivolumab and ipilimumab combination especially in patients in the middle and poor risk groups, and median survival was 26 months in the sunitinib 
group. However, for the patients in the good risk group, sunitinib clearly maintains its place in the first step (43).

PD-1 is a member of the CD 28 receptor family and they are expressed in naive and activated T, B-lymphocytes and natural killer (NK) cells in peripheral blood. PD-1 has two ligands, namely PD-L1 and PD-L2. When PD-1 binds to its ligand during recognition, it causes PD-1 to cross-link with the antigen receptor complex. Phosphorylation of the PD-1 receptor then leads to the recognition of SHP2, and inactivation of ZAP 70 in T cells and Syk in B cells (44). PD-1 activation induces inhibition of cell growth and cytokine secretion. It has also proven to be an important regulator in the immune response process and in the tolerance to environmental immunity. In tumor cells, it has been shown that PD-1 expression level increases, cytokine production is decreased and cytotoxicity of the tumor is impaired. Blockage of PD-1/PD-L1 pathway showed effective anti-tumor activity in Phase I/II studies $(45,46)$.

In Phase II IMmotion150 study comparing sunitinib with combination of atezolizumab and bevacizumab, PFS was found to be 7.8 months vs. 14.7 months, respectively, in $\mathrm{mRCC}$ patients with PD-L1 staining more than 1\% (47).

\section{Resistance Mechanisms Against Targeted Treatments in Renal Cell Carcinoma}

Studies have shown that there are two types of resistance to TKI treatment, including primary resistance and acquired resistance. Resistance to TKI treatment can be overcome by second-line treatments (mTOR, c-MET, PD-1 inhibitors or a combination of a mTOR inhibitor with a TKI).

\section{Primary Resistance}

The primary or intrinsic resistance mechanism is determined depending on the molecular properties of each tumor. Targeted therapies (VEGF-targeted inhibitors) have no clinical benefit for patients with this type of resistance. Gordan et al. (48) identified three groups of ccRCCs that were based on HIF- $\alpha$ detection and could explain intrinsic resistance: patients with detected HIF- $\alpha$ protein and wild-type VHL alleles, patients with VHL defective tumors (24\% methylation, 4\% homozygous deletion) and detected HIF- $1 \alpha$ and HIF- $2 \alpha$ expression, and patients with VHL defective tumors expressing HIF- $2 \alpha$ only (24\% methylation). These findings suggest that HIF- $1 \alpha$ and HIF- $2 \alpha$ promote different oncogene activation in ccRCC. The other mechanism presumed to cause primary resistance is the presence of proangiogenic signals that bypass the TKI inhibition and thus allow angiogenesis to continue (49). Other mechanisms associated with primary resistance include increased expression of $\mathrm{B}$ cell lymphoma-2 (Bcl-2) and/or $\mathrm{Bcl}-\mathrm{XL}$ proteins that are important in inhibition of apoptosis and a reduction in expression of CD95 (50).

Epigenetic modifications of histone protein in chromatin have been shown to play an important role in the regulation of gene transcription patterns in cells by the catalytic activity of histone deacetylase and methyltransferase. Changes in genes encoding these enzymes have been identified in RCC (36). In recent studies, it has been shown that inactivation of antiangiogenic factors by methylation of the histone methyltransferase at the promoter site of EZH2 contributes to tumor angiogenesis. EZH2 overexpression contributes to the development of resistance to TKI treatment (51).

\section{Conclusion}

\section{Acquired Resistance}

Resistance to TKI treatments may be possible with various mechanisms. As a result of various studies, it has been shown that the regulation of angiogenesis-related genes around the tumor, increasing the rate of pericyte cells in the vascular bed, removal of pro-angiogenic inflammatory cells from the bone marrow, or increasing the ability of tumor cells to invade healthy tissues are effective in acquired resistance in resistant RCCs (49). Despite the various mechanisms described for resistance to targeted-therapy, currently there are no biomarkers available to describe drug resistance in patients.

\section{Activation of Alternative Pro-angiogenic Pathways}

Activation of alternative pro-angiogenic pathways independent of VEGF after TKI treatment is one of the most common resistance mechanisms. The studies showed overexpression of pro-angiogenic factors such as fibroblast growth factor 1 and 2 (FGF1/2), IL-8, efrin A1 and A2 (Efna1/2) and angiopoietin 1 and 2 (Ang1/2) as a result of hypoxia induced by antiangiogenic therapy (52). FGF can directly stimulate endothelial cell proliferation and the formation of endothelial tubules in the presence of TKI. IL-8 is a pro-angiogenic factor with high expression in TKI-resistant patients, and its expression is regulated by the transcription factor NF- $\kappa B$ independent of the HIF-1 $\alpha$ pathway.

IL-8 binds to the CXCR2 receptor and leads to autocrine activation of VEGFR- 2 and consequently increased angiogenesis as a result of proliferation of VEGF $m R N A$ and its dependent protein expression in endothelial cells (53). Ang 2 expression significantly increases with hypoxia and loss of VHL gene in RCC. Ang 2 functions as a natural antagonist of Ang 1 and is only expressed in active angiogenesis, vascular remodeling, pathological angiogenesis processes in tumors (54). However, the function of Ang 2 may vary depending on other pro angiogenic signals.

Placental growth factor (PIGF) is a VEGF homolog which is expressed by tumor cells, endothelial cells, bone marrow-induced pro-angiogenic cells, inflammation cells, and stromal cells, and which binds to VEGFR-1. Binding of PIGF to VEGFR-1 stimulates angiogenesis. The PIGFs/VEGFR-1 complex strengthens the VEGFR-2 signals and thus increases angiogenesis. In addition, PIGF enhances the expression of VEGF-A, FGF2, PDGF $\beta$ and matrix metalloproteinases (MMPs) and stimulates angiogenesis through various mechanisms (54).

VEGF-targeted therapy may cause metabolic stress in cancer cells that are exposed to oxygen and nutrient deficiency as a result of angiogenesis inhibition. In response to this, cells activate alternative signaling pathways such as the PI3K/AKT/ mTOR pathway for amino acids and other energy sources required for protein synthesis, cell growth and proliferation. It has been suggested that activation of $P / 3 K / A K T / m T O R$ pathway 
correlates with aggressive RCC tumor behavior and poor prognosis $(55,56)$.

\section{Resistance Induced by Tumor Microenvironment}

Tumor stroma is composed of endothelial cells, fibroblasts, pericytes and hematopoietic cells. These cells play an important role in angiogenesis and tumorigenesis by directly contributing to vascularization or secreting angiogenic factors (VEGF and MMP-9). Tumor-associated fibroblasts play an active role in tumorigenesis and may develop resistance to antiangiogenic therapy with VEGF inhibitors, and increased PDGF-C expression plays a role in this resistance mechanism (57). Pericytes are another type of stromal cell that directly contributes to the formation of blood vessels. The binding of PDGF-BB in endothelial cells to PDGFR- $\beta$ in pericytes leads to increased VEGF mRNA transcription by MAPK and PI $K$ in pericytes, and induces the survival of endothelial cells in a paracrine way (58).

Increasing pericyte cell count and VEGF production may result in increased survival of endothelial cells, and may make endothelial cells less susceptible to VEGF inhibition signals. On the other hand, the reduction of pericytes and loss of function cause the loss of vessel stabilization, resulting in vascularization of tumor cells and thus facilitating metastasis (59). Because of hypoxia resulting from the regression of tumor vessels, pro-angiogenic inflammation cells, such as CD11b + Gr1 + myeloid-derived suppressor cells (MDSC), increase in the tumor microenvironment (60). These cells can also be added to tumor endothelium and differentiate into endothelial cells. Angiogenesis is activated independently of VEGF in the tumor as a result of a higher expression of the pro-angiogenic Bombina variegata factor in CD11b + Gr1 + MDSC in resistant tumors.

\section{Increased Invasive Feature and Metastasis}

It has been suggested that the ability of tumors to metastasize to other cells increases with tumor hypoxia. Hypoxia also stimulates the expression of c-MET receptors. c-MET activation leads to tumor cell proliferation, increased survival and increased invasiveness with various signaling pathways such as PI3K/AKT, MAPK, Src and STAT3 (61). Recently, increased activity of c-MET has been shown to increase epithelialmesenchymal transition (62). After a prolonged extracellular stimulation, the accumulation of protein in the cell leads to cellular changes and the epithelial cells are freed from their typical biological structures. The expression of molecules such as platelet endothelial cell adhesion molecule 1 (PECAM1/ CD31), homeobox A9 and endothelial cell-specific molecule 1 is reduced in these cells (63). Expression of MMPs increases and thus leads to polarization and deformation by allowing cell-cell adhesion and decreased cell penetration (63). Reduction of cellcell adhesion and increased expression of MMPs increase the invasion characteristics of cells to other tissues.

\section{Lysosomal Sequestration}

The preclinical study results showed that the concentration of sunitinib, known as intracellular TKI, was ten-fold higher in resistant cells than sensitive cells. The hydrophobic structure of sunitinib allows this molecule to easily pass through the lysosomal plasma membrane, but the acidic environment of the lysosome does not allow its release. These results support the idea that sunitinib is retained in lysosomes. This mechanism protects the cell against antiangiogenic activity despite its high intracellular concentration of sunitinib. This mechanism provides a new model for transient acquired resistance (64). Lysosomal sequestration as a resistance mechanism has been proven to be reversible (65).

\section{Single-nucleotide Polymorphisms}

Single nucleotide polymorphisms (SNPs) found in genes that regulate pharmacokinetics and pharmacodynamics of TKIs may play a role in the development of resistance to VEGF-targeted therapy (66). SNPs in NR1I2 and NR1I3 nuclear receptor genes may negatively affect PFS and/or OS by negatively regulating CYP3A4 expression. Single nucleotide polymorphisms with pharmacodynamic factors such as TKI targets (VEGFR and PDGFR) may also contribute to the development of resistance to TKI (67).

\section{Resistance Through MicroRNAs}

In microRNA (miRNA) profile studies, different miRNA patterns were identified in RCC. The expression of miRNA-942, miRNA133a, miRNA-628-5p and miRNA-484 was higher in TKI-resistant RCC tumors compared to TKI-sensitive tumors. Overexpression of miRNA-942 in an RCC cell line increased MMP-9 and VEGF release and caused the migration of endothelial cells and increased treatment resistance (68).

\section{Prevention of Resistance to Treatment in RCC}

There are different strategies to prevent and overcome resistance to TKIs in the treatment of RCC. These include transition to a different alternative drug (either a VEGF or mTOR targeted therapy) and combined therapies (69). The underlying mechanisms for poor results in patients with primary resistance are complex. Understanding and preventing these mechanisms is important. As a result of preclinical studies in this direction, the efficacy of trametinib, a MEK inhibitor drug developed against RAS/MEK/ERK and PI3K/AKT pathways, has not yet been demonstrated in metastatic RCC patients (70).

\section{Replacement with the Same Drug Group}

Treatment with a TKI and a different subsequent TKI gave positive results in patients with advanced RCC. This approach is based on the fact that different TKls have different target profiles and potential (71). Sunitinib targets multiple kinase receptors including VEGFR-1, 2 and 3, PDGFR- $\alpha$ and $\beta$, c-KIT, FLT-3, CSF-1R and RET. On the other hand, sorafenib inhibits targets found in tumor cell (CRAF, BRAF, V600E BRAF, C-KIT and FLT-3) and tumor vasculature (CRAF, VEGFR-2 and 3 and PDGFR$\beta)$ (72). In patients with metastatic RCC, the anti-tumor activity of axitinib has been shown after treatment failure with sorafenib and sunitinib (71).

\section{Ethics}

Peer-review: Externally peer-reviewed.

\section{Authorship Contributions}

Consept: Y.Ü., Design: S.R., Y.,Ü., Data Collection or Processing: S.R., H.A.Y., Analysis and/or Interpretation: S.R., Y.Ü., Literature Search: S.R., H.A.Y., Writing: S.R., H.A.Y., Ç.A. 
Conflict of Interest: No conflict of interest was declared by the authors.

Finacial Disclosure: The authors declared that this study received no financial support.

\section{References}

1. Siegel RL, Miller KD, Jemal A. Cancer statistics, 2018. CA Cancer J Clin 2018;68:7-30.

2. Li QK, Pavlovich CP, Zhang $\mathrm{H}$, et al. Challenges and opportunities in the proteomic characterization of clear cell renal cell carcinoma (cCRCC): A critical step towards the personalized care of renal cancers. Semin Cancer Biol 2018; doi: 10.1016/j.semcancer.2018.06.004.

3. Rini BI, Campbell SC, Escudier B. Renal cell carcinoma. Lancet 2009; 373:1119-1132.

4. Yap NY, Rajandram R, Ng KL, et al. Genetic and Chromosomal Aberrations and Their Clinical Significance in Renal Neoplasms. BioMed research international 2015;2015:476508.

5. Maher ER. Genomics and epigenomics of renal cell carcinoma. Seminars in Cancer Biology 2012;23:10-17.

6. Latif F, Tory K, Gnarra J, et al Identification of the von Hippel-Lindau disease tumor suppressor gene. Science 1993;260:1317-1320.

7. van Houwelingen KP, van Dijk BA, Hulsbergen-van de Kaa CA, et al. Prevalence of von Hippel-Lindau gene mutations in sporadic renal cell carcinoma: results from The Netherlands cohort study. BMC cancer 2005;5:57.

8. Moch $\mathrm{H}$, Schraml P, Bubendorf L, et al. Intratumoral heterogeneity of von Hippel-Lindau gene deletions in renal cell carcinoma detected by fluorescence in situ hybridization. Cancer Res 1998;58:2304-2309.

9. Clifford SC, Prowse AH, Affara NA, et al. Inactivation of the von Hippel-Lindau (VHL) tumour suppressor gene and allelic losses at chromosome arm $3 p$ in primary renal cell carcinoma: evidence for a VHL-independent pathway in clear cell renal tumourigenesis. Genes Chromosomes Cancer 1998;22:200-209.

10. Fishman MN. Targeted Therapy of Kidney Cancer: Keeping the Art Around the Algorithms. Cancer Control 2013;20:222-232.

11. Singh RB, Amare Kadam PS. Investigation of tumor suppressor genes apart from VHL on $3 p$ by deletion mapping in sporadic clear cell renal cell carcinoma (cRCC). Urol Oncol 2013;31:1333-1342.

12. Donninger $\mathrm{H}$, Clark JA, Monaghan MK, et al. Cell cycle restriction is more important than apoptosis induction for RASSF1A protein tumor suppression. J Biol Chem 2014; 289:31287-31295.

13. Yanagawa N, Tamura G, Oizumi H, et al. Promoter hypermethylation of RASSF1A and RUNX3 genes as an independent prognostic prediction marker in surgically resected non-small cell lung cancers. Lung Cancer 2007;58:131-138.

14. Morrissey $C$, Martinez A, Zatyka M, et al. Epigenetic inactivation of the RASSF1A 3p21.3 tumor suppressor gene in both clear cell and papillary renal cell carcinoma. Cancer Res 2001;61:7277-7281.

15. Duns G, Hofstra RM, Sietzema JG, et al. Targeted exome sequencing in clear cell renal cell carcinoma tumors suggests aberrant chromatin regulation as a crucial step in ccRCC development. Hum Mutat 2012; 33:1059-1062.

16. Lichner Z, Scorilas A, White NM, et al. The chromatin remodeling gene ARID1A is a new prognostic marker in clear cell renal cell carcinoma. Am J Pathol 2013;182:1163-1170.

17. Liao L, Testa JR, Yang H. The Roles of Chromatin-Remodelers and Epigenetic Modifiers in Kidney Cancer. Cancer Genet 2015;208:206-214.

18. Li J, Guo L, Ai Z. An integrated analysis of cancer genes in clear cell renal cell carcinoma. Future Oncol 2017; 13:715-725.

19. Guo H, German P, Bai S, et al. The PI3K/AKT Pathway and Renal Cell Carcinoma. J Genet Genomics 2015;42:343-353.
20. Sternberg CN, Davis ID, Mardiak J, et al. Pazopanib in locally advanced or metastatic renal cell carcinoma: results of a randomized phase III trial. J Clin Oncol 2010;28:1061-1068.

21. Sternberg CN, Hawkins RE, Wagstaff J, et al. A randomised, doubleblind phase III study of pazopanib in patients with advanced and/ or metastatic renal cell carcinoma: final overall survival results and safety update. Eur J Cancer 2013;49:1287-1296.

22. Motzer RJ, Hutson TE, Pharm D, et al. Sunitinib versus Interferon Alfa in Metastatic Renal-Cell Carcinoma. N Engl J Med 2007;356:115-124.

23. Motzer RJ, Hutson TE, Cella D, et al. Pazopanib versus sunitinib in metastatic renal-cell carcinoma. N Engl J Med 2013;369:722-731.

24. Motzer RJ, Hutson TE, McCann L, et al. Overall survival in renalcell carcinoma with pazopanib versus sunitinib. N Engl J Med 2014;370:1769-1770.

25. Escudier B, Pluzanska A, Koralewski $P$, et al. Bevacizumab plus interferon alfa-2a for treatment of metastatic renal cell carcinoma: a randomised, double-blind phase III trial. Lancet 2007; 370:2103-2111.

26. Dranitsaris G, Vincent MD, Yu J, et al. Development and validation of a prediction index for hand-foot skin reaction in cancer patients receiving sorafenib. Ann Oncol 2012;23:2103-2108.

27. Rini BI, Escudier B, Tomczak P, et al. Comparative effectiveness of axitinib versus sorafenib in advanced renal cell carcinoma (AXIS): a randomised phase 3 trial. Lancet 2011;378:1931-1939.

28. Hutson TE, Lesovoy V, Al-Shukri S, et al. Axitinib versus sorafenib as first-line therapy in patients with metastatic renal-cell carcinoma: a randomised open-label phase 3 trial. Lancet Oncol 2013;14:1287-1294.

29. Yakes FM, Chen J, Tan J, et al. Cabozantinib (XL184), a novel MET and VEGFR2 inhibitor, simultaneously suppresses metastasis, angiogenesis, and tumor growth. Mol Cancer Ther 2011;10:2298-2308.

30. Choueiri TK, Escudier B, Powles T, et al. Cabozantinib versus Everolimus in Advanced Renal-Cell Carcinoma. N Engl J Med 2015;373:1814-1823.

31. Choueiri TK, Escudier B, Powles T, et al. Cabozantinib versus everolimus in advanced renal cell carcinoma (METEOR): final results from a randomised, open-label, phase 3 trial. Lancet Oncol 2016;17:917-927.

32. Hasinoff BB, Wu X, Nitiss JL, et al. The anticancer multi-kinase inhibitor dovitinib also targets topoisomerase I and topoisomerase II. Biochem Pharmacol 2012; 84:1617-1626.

33. Motzer RJ, Hutson TE, Glen $H$, et al. Lenvatinib, everolimus, and the combination in patients with metastatic renal cell carcinoma: a randomised, phase 2, open-label, multicentre trial. Lancet Oncol 2015;16:1473-1482.

34. Motzer RJ, Hutson TE, Ren $M$, et al. Independent assessment of lenvatinib plus everolimus in patients with metastatic renal cell carcinoma. Lancet Oncol 2016; 17:e4-e5.

35. Faivre S, Kroemer G, Raymond E. Current development of mTOR inhibitors as anticancer agents. Nat Rev Drug Discov 2006;5:671-688.

36. Creigton CJ, Morgan M, Gunaratne PH, et al. Comprehensive molecular characterization of clear cell renal cell carcinoma. Nature 2013;499:43-49.

37. Figlin RA, Kaufmann I, Brechbiel J. Targeting PI3K and mTORC2 in metastatic renal cell carcinoma: New strategies for overcoming resistance to VEGFR and mTORC1 inhibitors. Int I Cancer 2013;133:788-796.

38. Hudes G, Carducci M, Tomczak P, et al. Temsirolimus, interferon alfa, or both for advanced renal-cell carcinoma. N Engl J Med 2007; 356:2271-2281.

39. Hutson TE, Escudier B, Esteban E, et al. Randomized Phase III Trial of Temsirolimus Versus Sorafenib As Second-Line Therapy After Sunitinib in Patients With Metastatic Renal Cell Carcinoma. J Clin Oncol 2014;32:760-767. 
40. Calvo E, Escudier B, Motzer RJ, et al. Everolimus in metastatic renal cell carcinoma: Subgroup analysis of patients with 1 or 2 previous vascular endothelial growth factor receptor-tyrosine kinase inhibitor therapies enrolled in the phase III RECORD-1 study. Eur J Cancer 2012;48:333-339.

41. George S, Motzer RJ, Hammers HJ, et al. Safety and Efficacy of Nivolumab in Patients With Metastatic Renal Cell Carcinoma Treated Beyond Progression: A Subgroup Analysis of a Randomized Clinical Trial. JAMA Oncol 2016;2:1179-1186.

42. Motzer RJ, Escudier B, McDermott DF, et al. Nivolumab versus Everolimus in Advanced Renal-Cell Carcinoma. N Engl J Med 2015;373:1803-1813.

43. Motzer RJ, Tannir NM, McDermott DF, et al. Nivolumab plus Ipilimumab versus Sunitinib in Advanced Renal-Cell Carcinoma. N Engl J Med 2018;378:1277-1290.

44. Parry RV, Chemnitz JM, Frauwirth KA, et al. CTLA-4 and PD-1 receptors inhibit T-cell activation by distinct mechanisms. Mol Cell Biol 2005;25:9543-9553.

45. Weber J. Immune checkpoint proteins: a new therapeutic paradigm for cancer--preclinical background: CTLA-4 and PD-1 blockade. Semin Oncol 2010;37:430-439.

46. Callahan MK, Wolchok JD. At the bedside: CTLA-4- and PD-1-blocking antibodies in cancer immunotherapy. J Leukoc Biol 2013;94:41-53.

47. Atkins MB, McDermott DF, Powles T, et al. IMmotion150: A phase II trial in untreated metastatic renal cell carcinoma (mRCC) patients (pts) of atezolizumab (atezo) and bevacizumab (bev) vs and following atezo or sunitinib (sun). Am Soc Clin Oncol 2017(suppl):4505-4505

48. Gordan JD, Lal P, Dondeti VR, et al. HIF-alpha effects on C-Myc distinguish two subtypes of sporadic VHL-deficient clear cell renal carcinoma. Cancer Cell 2008;14:435-446.

49. Bergers G, Hanahan D. Modes of resistance to anti-angiogenic therapy. Nat Rev Cancer 2008;8:592-603.

50. Gobé G, Rubin M, Williams G, et al. Apoptosis and expression of $\mathrm{BCl}-2, \mathrm{Bcl}-\mathrm{XL}$, and $\mathrm{Bax}$ in renal cell carcinomas. Cancer Invest 2002;20:324-332.

51. Adelaiye R, Ciamporcero E, Miles KM, et al. Sunitinib dose escalation overcomes transient resistance in clear cell renal cell carcinoma and is associated with epigenetic modifications. Mol Cancer Ther 2015;14:513-522.

52. Casanovas O, Hicklin DJ, Bergers G, et al. Drug resistance by evasion of antiangiogenic targeting of VEGF signaling in late-stage pancreatic islet tumors. Cancer Cell 2005;8:299-309.

53. Martin D, Galisteo R, Gutkind JS. CXCL8/IL8 stimulates vascular endothelial growth factor (VEGF) expression and the autocrine activation of VEGFR2 in endothelial cells by activating NFkappaB through the CBM (Carma3/Bcl10/Malt1) complex. J Biol Chem 2009;284:6038-6042.

54. Currie MJ, Gunningham SP, Turner K, et al. Expression of the angiopoietins and their receptor Tie2 in human renal clear cell carcinomas; regulation by the von Hippel-Lindau gene and hypoxia. J Pathol 2002;198:502-510.

55. Pantuck AJ, Seligson DB, Klatte T, et al. Prognostic relevance of the mTOR pathway in renal cell carcinoma: implications for molecular patient selection for targeted therapy. Cancer 2007;109:2257-2267.
56. Jones RG, Thompson CB. Tumor suppressors and cell metabolism: a recipe for cancer growth. Genes Dev 2009;23:537-548.

57. Crawford Y, Kasman I, Yu L, et al. PDGF-C mediates the angiogenic and tumorigenic properties of fibroblasts associated with tumors refractory to anti-VEGF treatment. Cancer Cell 2009;15:21-34.

58. Reinmuth N, Liu W, Jung YD, et al. Induction of VEGF in perivascular cells defines a potential paracrine mechanism for endothelial cell survival. FASEB J 2001;15:1239-1241.

59. Xian X, Håkansson J, Ståhlberg A, et al. Pericytes limit tumor cell metastasis. J Clin Invest 2006;116:642-651.

60. Yang L, DeBusk LM, Fukuda K, et al. Expansion of myeloid immune suppressor $\mathrm{Gr}+\mathrm{CD} 11 \mathrm{~b}+$ cells in tumor-bearing host directly promotes tumor angiogenesis. Cancer Cell 2004;6:409-421.

61. Shojaei F, Lee JH, Simmons BH, et al. HGF/c-Met acts as an alternative angiogenic pathway in sunitinib-resistant tumors. Cancer Res 2010;70:10090-10100.

62. Lu KV, Chang JP, Parachoniak CA, et al. VEGF inhibits tumor cell invasion and mesenchymal transition through a MET/VEGFR2 complex. Cancer Cell 2012; 22:21-35.

63. Arao T, Matsumoto K, Furuta K, et al. Acquired drug resistance to vascular endothelial growth factor receptor 2 tyrosine kinase inhibitor in human vascular endothelial cells. Anticancer Res 2011;31:2787-2796.

64. Gotink KJ, Broxterman HJ, Labots M, et al. Lysosomal sequestration of sunitinib: a novel mechanism of drug resistance. Clin Cancer Res 2011;17:7337-7346.

65. Grünwald V, Weikert S, Seidel C, et al. Efficacy of sunitinib re-exposure after failure of an mTOR inhibitor in patients with metastatic RCC. Onkologie 2011;34:310-314.

66. van der Veldt AA, Eechoute K, Gelderblom H, et al. Genetic polymorphisms associated with a prolonged progression-free survival in patients with metastatic renal cell cancer treated with sunitinib. Clin Cancer Res 2011;17:620-629.

67. Beuselinck B, Karadimou A, Lambrechts D, et al. VEGFR1 single nucleotide polymorphisms associated with outcome in patients with metastatic renal cell carcinoma treated with sunitinib - a multicentric retrospective analysis. Acta Oncol 2014;53:103-112.

68. Prior C, Perez-Gracia JL, Garcia-Donas J, et al. Identification of tissue microRNAs predictive of sunitinib activity in patients with metastatic renal cell carcinoma. PLoS One 2014;9:e86263.

69. Rini BI, Wilding G, Hudes G, et al. Phase II study of axitinib in sorafenib-refractory metastatic renal cell carcinoma. J Clin Oncol 2009;27:4462-4468.

70. Bridgeman VL, Wan E, Foo S, et al. Preclinical Evidence That Trametinib Enhances the Response to Antiangiogenic Tyrosine Kinase Inhibitors in Renal Cell Carcinoma. Mol Cancer Ther 2016;15:172-83.

71. Broekman F, Giovannetti E, Peters G]. Tyrosine kinase inhibitors: Multi-targeted or single-targeted? World J Clin Oncol 2011;2:80-93.

72. Schmid TA, Gore ME, Sunitinib in the treatment of metastatic renal cell carcinoma. Ther Adv Urol 2016;8:348-371. 